\title{
Recovery and detachment between shifts, and fatigue during a twelve-hour shift
}

\author{
Christian Korunka ${ }^{*}$, Bettina Kubicek, Roman Prem and Antonio Cvitan \\ Faculty of Psychology, University of Vienna, Austria
}

\begin{abstract}
The study investigated the role of recovery and detachment in the break period between two shifts for fatigue in the current shift. A time-based paper-and-pencil diary study was carried out observing sixty-four railway controllers over ten consecutive working shifts. The results demonstrated that fatigue in the current shift was not only affected by recovery and psychological detachment during break phases before a shift, but also by fatigue at shift onset and perceived workload during the shift.
\end{abstract}

Keywords: Shift work, diary study, recovery, psychological detachment, fatigue

\section{Introduction}

Recent meta-analyses confirm negative impacts of shift work on mental health outcomes [20]. Shift work in general, but especially night-shift work, presents a significant problem with regard to wellbeing, health, and occupational safety. In contrast to many other potential work-related design factors which may be harmful to health at the workplace, shift work generally cannot be avoided. Shift work is increasingly demanded to provide services around the clock [6].

From a macro-ergonomic perspective, shiftwork constitutes a specific challenge because of the immanent constraints of this specific type of work in improving the working conditions itself. For instance, night work and irregular working times are inherent characteristics of shift work; they cannot generally be avoided. On the other hand, the succession and length of shift and break periods could be designed based on macro-ergonomic criteria. Thus, the design of shift rosters, and especially the break period between shifts and recovery quality may be of especial importance for reducing fatigue and improving wellbeing and occupational safety during shift work. Many studies were published dealing with the effects of different shift lengths [16]. Most of these studies confirm the advantages of twelve-hour shift systems compared with shorter shift periods [1]. There is general agreement that, when designing shift rosters with the goal of improving employee well-being and mental health, the psychosocial working conditions of employees (age, gender, family status, commuting time to the work place, etc.) need to be taken into consideration.

Compared to the well-analyzed effects of shift lengths and shift rosters, relatively little is known about the role and importance of recovery times and recovery quality for fatigue and well-being during a subsequent shift. Recovery is the process that reverses the negative consequences of job demands and brings an individual back to his or her pre-stressor functioning [3]. In periods of recovery, employees are no longer confronted with work-related demands and are able to rebuild resources.

In recent years, research has focused on detachment during recovery periods as a facilitating process for a successful recovery process [17]. Psychological detachment refers to an "individual's sense of being away from the work situation" [4]. It was found that lack of psychological detachment from work during recovery periods is related to impaired psychological well-being [17]. On the other hand, detachment from work during recovery correlates positively with in-

\footnotetext{
*Address for correspondence: Prof. Christian Korunka, Ph.D., Faculty of Psychology, University of Vienna, Universitätsstraße 7, A-1010
} Vienna, Austria. Tel.: +43 14277 47881; Fax: +43 14277 9473; E-mail: christian.korunka@univie.ac.at. 
creased work engagement and reduced strain. The recovery value and the quality of detachment from work during a break period are likely to vary as a function of its duration and the time at which it occurs [18].

Thus one may expect both recovery and detachment processes between working shifts to exert a moderating effect on the workload-fatigue relationship in the current shift. Perceived workload in the current shift may also affect fatigue.

An important requirement of any studies aimed at analyzing the effects of the previous shift's workload, and of recovery and detachment processes between shifts, on well-being in current working shifts, is that they be designed longitudinally, with a defined number of measuring points, both within and between working shifts. During the past decade, diary studies have been increasingly used in organizational research because they meet such methodological criteria perfectly [11]. One advantage of diaries, as compared to traditional survey studies, is that data can be collected at the daily level and even several times a day, thereby "capturing life as it is lived" [2]. Diary studies may be event-based to capture the effects of specific triggering events or time-based to measure effects at fixed or random time intervals. Thus, diary studies are an ideal methodological approach for implementing a process perspective in macroergonomic research.

The current study is designed as a time-based diary study testing the following hypotheses:

(1) Recovery and detachment in a break period before the current shift affects fatigue in the current shift.

(2) Perceived load affects fatigue in the current shift.

(3) Recovery moderates the effects of perceived load on fatigue in the current shift.

(4) Detachment moderates the effects of perceived load on fatigue in the current shift.

\section{Method}

\subsection{Procedure and participants}

In April and May of 2010 a time-based paper-andpencil diary study [2] was conducted at a fully computerized central railway control center in Central Europe. In this center, participating signalers and controllers (both subsequently referred to as "controllers") $[5,13]$ were monitoring and controlling rail traffic in an area of approximately $12,500 \mathrm{~km}^{2}$ around the clock in twelve-hour shifts.

Each participant was given a questionnaire and a diary that was to be kept over a period of ten consecutive working shifts. The six-page questionnaire contained items regarding socio-demographic information; it was immediately filled out and collected. To protect participant privacy, the four diary entries for each of the ten shifts were made on separate sheets, perforated so as to be easily torn out of the diary upon completion, put in an envelope and mailed to the researchers after the shift.

The diary entry at shift onset contained scales for the current level of fatigue, as well as for recovery and psychological detachment during the previous break period. The remaining three diary entries were made every four hours thereafter (four, eight and twelve hours after shift onset), collecting data about current fatigue, perceived workload, and perceived time control during the preceding four hours.

From a total of seventy-one employees working at the railway control center at the time the diary study was conducted, sixty-four participated voluntarily in the study (return rate 90\%). All sixty-four respondents were male. As compared with the general working population, participants' average age $(M=47.11$ years, $S D=4.94)$ and average job tenure $(M=26.51$ years, $S D=6.73)$ were relatively high. Sixty of the sixty-four participants provided diary entries over ten full consecutive shifts with the remaining four participants filling out at least four consecutive shifts, resulting in a total of 626 diary data sets.

The controllers at the railway control center were members either of one of four shift groups working according to a main shift roster or of a fifth shift group with a less regular sequential arrangement of working shifts. Within the main shift roster, a 12-hr day shift was followed by a $24-\mathrm{hr}$ break before a 12 hr night shift, this was followed by a 48 -hr break before the cycle began anew with a 12-hr day shift. Because of this sequence of shifts on the main shift roster, approximately two-thirds of the diary data sets had one of these sequences:

(1) 24-hr break period - 12-hr night shift $(n=223$ diary data sets);

(2) 48-hr break period - 12-hr day shift $(n=185$ diary data sets).

Thus the hypotheses had to be tested separately for these two shift rosters. 


\subsection{Diary measures}

Fatigue. Fatigue was measured at shift onset and every four hours thereafter using strain ratings (Beanspruchungsratings), a single item adopted from a German scale designed to capture consequences of strain in diary studies [15]. To obtain the level of fatigue current at the points in time when diary entries were completed, participants were asked to indicate how tired they felt at that moment on a 6 -point rating scale $(1=$ not at all, and $6=$ a lot $)$.

Recovery. At shift onset, recovery during the previous break period was assessed using a three-item scale; the items it was based on were originally developed to measure its antipode, need for recovery, in diary studies [19].

Using a 5-point rating scale $(1=$ not at all true, and $5=$ utterly true) participants indicated whether: (a) "During the time off I would have needed more time for relaxing and recovering from work" (recoded); (b) "Considering the total of all activities that I pursued during my time off, I have had enough time to relax and to recover from work today"; and (c) "All things considered I was able to recover sufficiently during the time off' (Cronbach's $\alpha=.89$ ).

Psychological detachment. The scale for recovery at shift onset was directly followed by a three-item scale measuring psychological detachment during the previous break period; here the items were adapted from a scale by Sonnentag and Bayer [17]. Using the same five-point rating scale as for recovery $(1=$ not at all true, and $5=$ utterly true), participants indicated whether: (a) "During my time off I forgot completely about my working day"; (b) "During my time off I could 'switch off' completely"; and (c) "During my time off I had to think about my work again and again" (recoded) (Cronbach's $\alpha=.86$ ).

Workload. The diary entries four, eight, and twelve hours after shift onset contained self-designed items measuring perceived workload. Monitoring and controlling the rail infrastructure requires mental workload to: (a) "be aware and assess the current situation"; (b) "make decisions and plan strategies to deal with [the] situation"; and (c) "act to implement [the] plan" [13]. To develop a plan it is often necessary to consult or negotiate with colleagues; to implement it safely, radio communication with drivers may be necessary. Monitoring, intervening and communicating are therefore core tasks for controllers. A scale was constructed to assess the workload levels of each of these predominant tasks, with seven rating points ( $1=$ very low, and $7=$ very high $)$, for the four-hour shift directly before the current diary entry. This three-item scale proved to have good internal consistency: Cronbach's $\alpha=.89$ after four hours, .91 after eight hours, and .92 after twelve hours of shift work.

Time control. The diary entries at four, eight, and twelve hours after shift onset also contained a selfdesigned item to assess time control, which was understood as a positive antipode of time pressure, and expected to be a resource for controllers while performing their job. Participants were asked to indicate the level of time control, using a seven-point rating scale $(1=$ very low, and $7=$ very high $)$, during the four-hour shift directly before the current diary entry.

\subsection{Data analysis procedure}

Given the clustering of measurement occasions (Level 1) within persons (Level 2), the data was analyzed using hierarchical linear modeling [8]. In order to reduce multicollinearity, Level 1 and Level 2 variables were grand-mean centered [7]. All analyses were conducted using MPlus 5.0 [10] and were performed separately for the two shift rosters. The dependent variables were; fatigue after four, eight, and twelve hours of shift work, resulting in six regression analyses.

In each of these regression analyses, an interceptonly model (null model) was estimated first. Then person-level (age, tenure) and day-level (fatigue at shift onset, time control in the current shift) control variables were entered into the regression equation (first model). In the second model, recovery and psychological detachment during the break period were added to test Hypothesis 1. Perceived workload in the current shift was entered in the third model (Hypothesis 2). The within-level interactions addressed in Hypotheses 3 and 4 were added in the fourth model. For brevity, only parameter estimates and standard errors from the last (fourth) model are listed in the results section. It is important to note, however, that estimates and standard errors showed only minor variations across models. 
Table 1

Results from hierarchical regression analyses predicting fatigue after 4, 8, and 12 hours of shift work

\begin{tabular}{|c|c|c|c|c|c|c|c|c|c|c|c|c|}
\hline \multirow[b]{3}{*}{ Variable } & \multicolumn{6}{|c|}{ Fatigue during night shifts } & \multicolumn{6}{|c|}{ Fatigue during day shifts } \\
\hline & \multicolumn{2}{|c|}{$\begin{array}{c}\text { After } 4 \mathrm{hrs} \\
(n=210)\end{array}$} & \multicolumn{2}{|c|}{$\begin{array}{c}\text { After } 8 \mathrm{hrs} \\
(n=221)\end{array}$} & \multicolumn{2}{|c|}{$\begin{array}{c}\text { After } 12 \mathrm{hrs} \\
(n=223)\end{array}$} & \multicolumn{2}{|c|}{$\begin{array}{l}\text { After } 4 \text { hrs } \\
(n=185)\end{array}$} & \multicolumn{2}{|c|}{$\begin{array}{c}\text { After } 8 \mathrm{hrs} \\
(n=184)\end{array}$} & \multicolumn{2}{|c|}{$\begin{array}{c}\text { After } 12 \mathrm{hrs} \\
(n=183)\end{array}$} \\
\hline & Est. & $S E$ & Est. & $S E$ & Est. & $S E$ & Est. & $S E$ & Est. & $S E$ & Est. & $S E$ \\
\hline Age & -.09 & .03 & -.45 & .05 & -.05 & .06 & .43 & .03 & .73 & .04 & $.87 *$ & .05 \\
\hline Tenure & .21 & .02 & .34 & .04 & -.02 & .04 & -.21 & .02 & -.43 & .04 & -.59 & .05 \\
\hline Fatigue at shift onset & $.59 * *$ & .08 & $.28 *$ & .08 & $.19 *$ & .07 & $.57 * *$ & .08 & $.59 * *$ & .08 & $.44 * *$ & .10 \\
\hline Time control in current shift & $.14^{*}$ & .04 & -.04 & .07 & -.12 & .09 & -.02 & .06 & .06 & .09 & .04 & .09 \\
\hline Recovery & $-.14 * *$ & .07 & -.03 & .10 & -.13 & .13 & -.18 & .10 & -.12 & .11 & $-.22 *$ & .12 \\
\hline Detachment & $-.14 *$ & .07 & -.09 & .10 & -.11 & .12 & -.02 & .09 & -.08 & .08 & $-.17 *$ & .11 \\
\hline Perceived workload in current shift & $.13 \dagger$ & .06 & -.04 & .07 & -.08 & .09 & .06 & .06 & $.19^{*}$ & .08 & $.22 *$ & .10 \\
\hline Perceived workload $*$ Recovery & $.12 *$ & .04 & $.17^{*}$ & .05 & .05 & .07 & .02 & .06 & .08 & .07 & .02 & .08 \\
\hline Perceived workload * Detachment & $-.14 *$ & .05 & .02 & .06 & .03 & .08 & -.05 & .04 & -.01 & .08 & .01 & .10 \\
\hline $\mathrm{R}^{2}$ (within level) & \multicolumn{2}{|c|}{$.55^{* *}$} & \multicolumn{2}{|c|}{$.13 \dagger$} & \multicolumn{2}{|c|}{.11} & \multicolumn{2}{|c|}{$.45^{* *}$} & \multicolumn{2}{|c|}{$.47 * *$} & \multicolumn{2}{|c|}{$.47 * *$} \\
\hline $\mathrm{R}^{2}$ (between level) & \multicolumn{2}{|c|}{.02} & \multicolumn{2}{|c|}{.05} & \multicolumn{2}{|c|}{.01} & \multicolumn{2}{|c|}{.07} & \multicolumn{2}{|c|}{.17} & \multicolumn{2}{|c|}{$.20 \dagger$} \\
\hline$-2 *$ Loglikelihood (Deviance) & \multicolumn{2}{|c|}{491.71} & \multicolumn{2}{|c|}{603.38} & \multicolumn{2}{|c|}{657.74} & \multicolumn{2}{|c|}{467.00} & \multicolumn{2}{|c|}{485.56} & \multicolumn{2}{|c|}{518.65} \\
\hline
\end{tabular}

Note. Est. $=$ standardized estimate; $S E=$ standard error; $\dagger p<.10 ; * p<.05 ; * * p<.01$.

\section{Results}

\subsection{Descriptive statistics}

In the course of night shifts, $t(2,464)=278.54$, $p<.01$, as well as of day shifts, $t(2,540)=110.66$, $p<.01$, controllers reported an increase in fatigue. While after four hours of nightly shift work, levels of fatigue were, on average, quite low $(M=2.47)$, they rose as high as 3.59 after eight hours and as high as 4.33 after twelve hours of shift work. During daytime shift work, average levels of fatigue were 2.36 (after $4 \mathrm{hrs}$ ), 2.92 (after $8 \mathrm{hrs}$ ), and 3.37 (after $12 \mathrm{hrs}$ ), respectively.

\subsection{Predicting fatigue during twelve-hour night shifts}

Before testing the study hypotheses, within- and between-persons variation in fatigue during night shifts were compared. Partitioning of the total variance revealed that $52 \%$ of the variance in fatigue after four hours of shift work was attributable to within-person variation. For fatigue after eight and twelve hours of shift work, $38 \%$ of the variance was within persons. These findings imply that a nontrivial proportion of the variance in fatigue during twelve hours of nightly shift work can be attributed to within-person variation.
For fatigue after four hours of nightly shift work as a dependent variable, including day-level recovery and psychological detachment in the analyses resulted in an improved model fit $(\Delta$ Deviance $=12.09$, $\Delta d f=2, p<.01)$, as did the inclusion of the interaction terms $(\Delta$ Deviance $=6.74, \Delta d f=2, p<.05)$. Recovery and psychological detachment during the previous break period were negatively related to fatigue after four hours of shift work, when age, tenure, time control and fatigue at the onset of the shift were controlled for. Moreover, these two variables qualified the effect of perceived workload on fatigue (see Table 1). Fig. 1 reveals that high levels of recovery counteracted fatigue only when perceived workload was low or average. Psychological detachment, on the other hand, clearly reduced the negative effect of high levels of perceived workload on controllers' fatigue after four hours of shift work (see Fig. 2).

After eight hours of nightly shift work, the only variable that improved the prediction of controller fatigue was the interaction between perceived workload and recovery $(\Delta$ Deviance $=4.82, \Delta d f=2, p<.10)$. As illustrated in Fig. 3, recovery buffered the negative effect of low workload on fatigue, suggesting that adequate off-the-job recovery helped controllers stay vigilant when workload was lacking during night shifts.

For fatigue after twelve hours of nightly shift work, including the predictor variables did not result in an improved model fit. Only the baseline levels of fatigue showed a significant positive association with fatigue at the end of the night shift. 


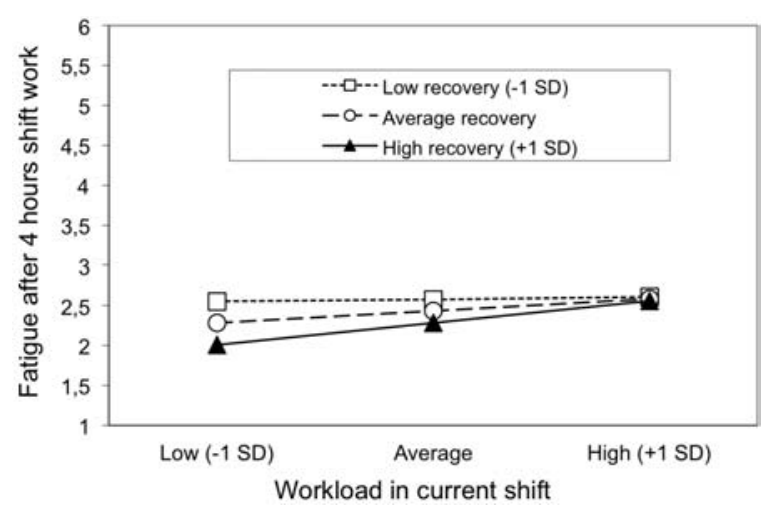

Fig. 1. Plot of the interaction between workload and recovery in predicting fatigue after 4 hours of nightly shift work

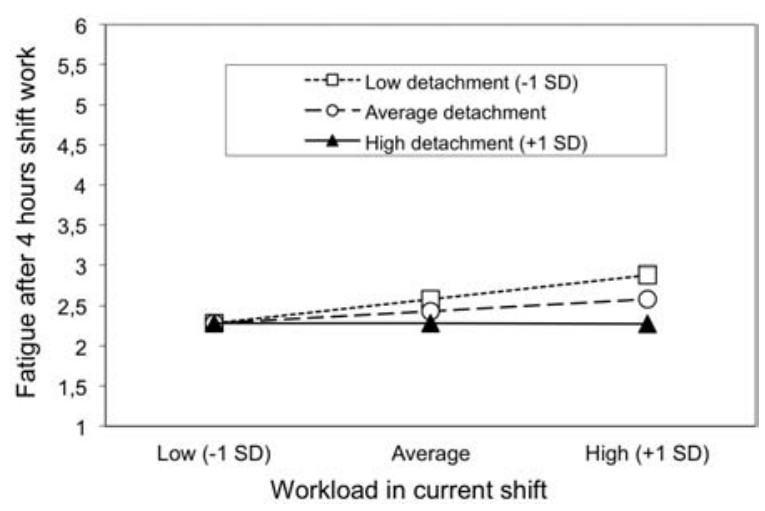

Fig. 2. Plot of the interaction between workload and detachment in predicting fatigue after 4 hours of nightly shift work

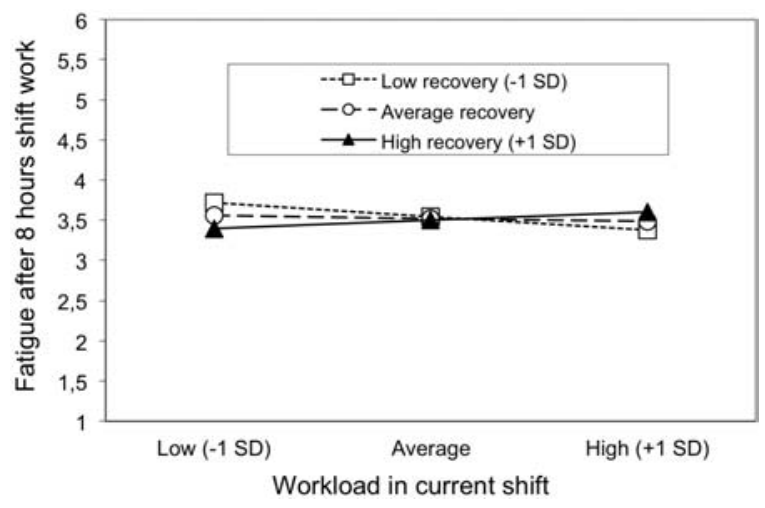

Fig. 3. Plot of the interaction between workload and recovery in predicting fatigue after 8 hours of nightly shift work

\subsection{Predicting fatigue during twelve-hour day shifts}

Initially, as for night shifts, the total variance in the dependent variable was partitioned into its between- and within-person components. For fatigue after four, eight and twelve hours of daytime shift work, $60 \%, 58 \%$ and $43 \%$ of the total variance were within persons, respectively. Thus, a substantial proportion of the variance in fatigue during day shifts can be explained at the within-person level.

For fatigue after four hours of daytime shift work as a dependent variable, including predictor variables and interaction terms did not cause a significant improvement of model fit. Rather, fatigue at the onset of the day shift was found to be the only significant predictor of the outcome variable (see right part of Table 1).

After eight hours of daytime shift work, the prediction of fatigue was improved by taking perceived workload into account $(\Delta$ Deviance $=5.84, \Delta d f=1$, $p<.05)$. The higher the perceived workload, the more weary controllers reported to be, irrespective of fatigue at shift onset.

Finally, after twelve hours of daytime shift work, the influences of fatigue at shift start and of perceived workload were supplemented by a significant age effect and by effects of recovery and psychological detachment $(\Delta$ Deviance $=14.96, \quad \Delta d f=2$, $p<.01)$. Older controllers and those reporting higher fatigue, lower recovery and lower psychological detachment at the beginning of the shift, as well as those reporting higher workload during the shift, were more tired at the end of a twelve-hour day of shift work (see Table 1).

\subsection{Summary of study results}

In sum, the influence of the predictor variables on fatigue was seen to accrue in the course of the day shifts and fade away in the course of the night shifts, leaving baseline levels of fatigue as the only important predictor of fatigue at the end of a night shift. Recovery, psychological detachment, and the interactions of these variables with workload predicted fatigue only after four hours of nightly shift work and not after eight or twelve hours. In daytime, perceived workload did not flow on to affect fatigue until eight hours of shift work; recovery and psychological detachment only revealed their effects after twelve hours of shift work. 


\section{Discussion}

This time-based diary study was designed to test the effects of recovery, psychological detachment, and workload on fatigue in the course of twelve-hour shifts. By taking a closer look at the antecedents of fatigue after four, eight and twelve hours of shift work, it contributes to an understanding of how these factors additively and interactively impact on employee strain. Due to differing lengths for the break periods after day shifts and night shifts, these two shift rosters were examined separately.

Overall, the study results supported not only the hypothesized restorative effect of recovery and psychological detachment, but also the assumed wearying effect of perceived workload. Yet, depending on the shift roster, these factors unfurled their influence on employee fatigue at different points in time. In day shifts that were anteceded by forty-eight-hour breaks, workload did not display its wearying effect before eight hours of shift work; adequate recovery and psychological detachment reduced fatigue only after twelve hours on day shift. In night shifts that were preceded by twenty-four-hour breaks, recovery and psychological detachment, as well as their interactions with workload, affected employee fatigue immediately, i.e., within the first four hours of the shift. Afterwards, fatigue at shift onset remained the only significant predictor of fatigue at shift's end.

In concordance with previous research [17, 19], the study results suggest that both psychological detachment and recovery are important off-the-job inhibitors of fatigue. Given the equally strong effects both of these variables had on employee strain, one might even argue that psychological detachment constitutes an integral part of recovery during break times [17].

In addition to their additive effects, recovery and detachment moderated the relationship between workload and fatigue in night shifts. While psychological detachment during break periods clearly reduced the negative effect of workload on fatigue, adequate recovery helped rail traffic controllers to fight fatigue when workload was low during night shifts. The latter finding suggests that off-the-job recovery facilitates maintaining vigilance under lowdemand conditions in monitoring tasks.

\subsection{Implications}

From a practical perspective, the study findings have implications for designing shift patterns that are beneficial for employee well-being. The tremendous rise in fatigue during nightly shift work found in this study suggests that shift length should not exceed twelve hours or should even be reduced to a maximum of eight hours. Moreover it seems advisable for organizations to design monitoring tasks in such a way that they prevent employees from experiencing monotony and low demands. This may be accomplished by adding additional tasks that are not timecritical and allowing employees to gain "increased control over the work process as well as opportunities for on-the-job training" [12], as indicated by previous studies.

From a methodological and theoretical perspective, the results suggest that the timing of, and time lag between, the measurements influence the relationship between predictor and outcome variables. This imposes a new challenge on theory development and testing in macro-ergonomic research: it requires considering the role of time in strain phenomena [9]. In conceptual models as well as in research design, time orders and intervals need to be modeled and planned carefully. One needs examine both how long after onset of the antecedent, and under which conditions, the outcome is affected.

\subsection{Strengths and limitations}

Time-based diaries are excellent tools for assessing the causal effects of on- and off-the-job factors on employee strain during shift work. They allow not only for the temporal separation required to test causation [14], but also for the identification of the time lag between outcomes and their antecedents.

The second strength of diary studies - to capture life as it is lived [2] - simultaneously constitutes a limitation in the present study. As in many field studies, the shift rosters were predetermined and not open to change. The different length of break periods after day and night shifts made it necessary to examine the shift rosters separately. This may limit opportunities to generalize the results to other shift patterns.

In addition to the specific shift and break lengths, the age composition of this sample may also impose limitations on the generalizability of the study results. The average age of the rail traffic controllers investigated in the present study was relatively high. This may have caused stronger effects of day-level recovery, psychological detachment and workload on fatigue in the present sample as compared to younger employees. Hence, when assessing the study results, 
the specific sample characteristics have to be kept in mind,.

\subsection{Conclusions}

Bringing together research on shift rosters and recreation processes, this study revealed that the effects of workload, recovery, and detachment on fatigue devolve differently during night and day shifts. While recovery and psychological detachment were important proximal predictors of fatigue after four hours of nightly shift work - but not after eight or twelve hours - they showed a lagged effect on fatigue in a daytime shift. The effect of perceived workload kicked in after eight hours of shift work; the influence of recovery and psychological detachment did not take effect before twelve hours of daytime shift work. Thus, alongside off-the-job recovery, reducing monotony in monitoring tasks during night shifts and decreasing workload during daytime shifts seem important in helping controllers fight fatigue.

\section{References}

[1] S.D. Baulk, A. Fletcher, K.J. Kandelaars, D. Dawson, and G.D. Roach, A field study of sleep and fatigue in a regular rotating 12-h shift system, Applied Ergonomics 40 (2009), 694698.

[2] N. Bolger, A. Davis, and E. Rafaeli, Diary methods: Capturing life as it is lived, Annual Review of Psychology 54 (2003), 579-616.

[3] A. Craig and R.E. Cooper, Symptoms of acute and chronic fatigue, in: Handbook of human performance, A.P. Smith and D.M. Jones, eds., Academic Press, London, 1992, pp. 289-339.

[4] D. Etzion, D. Eden, and Y. Lapidot, Relief from job stressors and burnout: Reserve service as a respite, Journal of Applied Psychology 4 (1998), 577-585.

[5] T. Farrington-Darby, J.R. Wilson, B.J. Norris, and T. Clarke, A naturalistic study of railway controllers, Ergonomics 49 (2006), 1370-1394.

[6] M.I. Härmä and J.E. Ilmarinen, Towards the 24-hour society new approaches for aging shift workers?, Scandinavian Journal of Work, Environment \& Health 25 (1999), 610-615.
[7] D.A. Hofmann and M.B. Gavin, Centering decisions in hierarchical linear models: Implications for research in organizations, Journal of Management 24 (1998), 623-641.

[8] J.J. Hox, Multilevel analysis. Techniques and applications, Routledge, New York, 2010.

[9] T.R. Mitchell and L.R. James, Building better theory: Time and the specification of when things happen, Academy of Management Review 26 (2001), 530-547.

[10]L.K. Muthén and B.O. Muthén, Mplus User's Guide, Muthén \& Muthén, Los Angeles, CA, 1998-2007.

[11]S. Ohly, S. Sonnentag, C. Niessen, and D. Zapf, Diary studies in organizational research: An introduction and some practical recommendations, Journal of Personnel Psychology 9 (2010), 79-93.

[12]A. Persson, B. Wanek, and A. Johansson, Passive versus active operator work in automated process control - a job design case study in a control centre, Applied Ergonomics 32 (2001), 441-451.

[13]L. Pickup, J.R. Wilson, S. Sharples, B. Norris, T. Clarke, and M.S. Young, Fundamental examination of mental workload in the rail industry, Theoretical Issues in Ergonomics Science 6 (2005), 463-482.

[14]P.M. Podsakoff, S.B. MacKenzie, and N.P. Podsakoff, Common method biases in behavioral research: A critical review of the literature and recommended remedies, Journal of Applied Psychology 88 (2003), 879-903.

[15]P. Richter, U. Debitz, and F. Schulze, Diagnostik von Arbeitsanforderungen und kumulativen Beanspruchungsfolgen am Beispiel eines Call Centers, Zeitschrift für Arbeitswissenschaft 56 (2002), 67-76.

[16]L. Smith, S. Folkard, P. Tucker, and I. Macdonald, Work shift duration: A review comparing eight hour and 12 hour shift systems, Occupational and Environmental Medicine 55 (1998), 217-229.

[17] S. Sonnentag and U.-V. Bayer, Switching off mentally: Predictors and consequences of psychological detachment from work during off-job time, Journal of Occupational Health Psychology 10 (2005), 393-414.

[18] S. Sonnentag, C. Binnewies, and E.J. Mojza, Staying well and engaged when demands are high: The role of psychological detachment, Journal of Applied Psychology 95 (2010), 965976.

[19] S. Sonnentag and F.R.H. Zijlstra, Job characteristics and offjob activities as predictors of need for recovery, well-being, and fatigue, Journal of Applied Psychology 91 (2006), 330350 .

[20] P. Tucker and S.R. Knowles, Review of studies that have used the Standard Shiftwork Index: Evidence for the underlying model of shiftwork and health, Applied Ergonomics 39 (2008), 550-564. 